HERDER JAHRBUCH

HERDER YEARBOOK

1998 


\section{Beirat}

Martin Bollacher, Ruhr-Universität Bochum

Richard Critchfield, Texas A \& M University

Otto Dann, Universität Köln

Gonthier-Louis Fink, Université de Strasbourg

Ulrich Gaier, Universität Konstanz

Hans Dietrich Irmscher, Universität Köln

Eva Knodt, Stanford, California

Wilfried Malsch, University of Massachusetts, Amherst

Karl Menges, University of California, Davis

Michael Morton, Duke University

Kurt Mueller-Vollmer, Stanford University

Hugh Barr Nisber, Sussex College Cambridge

Regine Otto, Stiftung Weimarer Klassik

Peter Hanns Reill, University of California, Los Angeles

Kurt Rudolph, Universität Marburg

Wilhelm Schmidt-Biggemann, Freie Universität Berlin

Helmut Schneider, Universität Bonn

Josef Simon, Universität Bonn

Jürgen Trabant, Freie Universität Berlin 


\title{
HERDER JAHRBUCH HERDER YEARBOOK 1998
}

\author{
Herausgegeben von \\ Hans Adler und Wulf Koepke \\ mit Samson B. Knoll als \\ Gastherausgeber
}

VERLAG J. B. METZLER

STUTTGART · WEIMAR 
Die Deutsche Bibliothek - CIP-Einheitsaufnahme

Herder-Jahrbuch $=$ Herder yearbook. - Stuttgart ; Weimar : Metzler. Erscheint jährl. - Früher im Verl. Camden House, Columbia SC. -

Wurde früher nicht angezeigt. - Aufnahme nach 1997

NE: Herder yearbook

ISSN 0948-5279

ISBN 978-3-476-01634-8

ISBN 978-3-476-03760-2 (eBook)

DOI.10.1007/978-3-476-03760-2

Dieses Werk einschließlich aller seiner Teile ist urheberrechtlich geschützt.

Jede Verwertung außerhalb der engen Grenzen des Urheberrechtsgesetzes ist ohne Zustimmung des Verlages unzulässig und strafbar. Das gilt insbesondere für Vervielfältigungen, Übersetzungen, Mikroverfilmungen und die Einspeicherung und Verarbeitung in elektronischen Systemen.

(C) 1998 Springer-Verlag GmbH Deutschland

Ursprünglich erschienen bei J.B. Metzlersche Verlagsbuchandlung

und Carl Ernst Poeschel Verlag GmbH in Stuttgart 1998 


\section{INHALT}

Vorwort der Herausgeber $\ldots \ldots \ldots \ldots \ldots \ldots \ldots \ldots \ldots \ldots \ldots \ldots . . \ldots \ldots \ldots$

JoHn H. Zammito (Houston, Texas): "Method" versus "Manner«?

Kant's Critique of Herder's Ideen in the Light of the Epoch

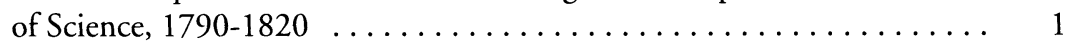

RALF SimON (Bonn): Apokalyptische Hermeneutik. Johann Gottfried Herder: Maran Atha, Geschichtsphilosophie, Adrastea . . 27

VICKI SPENCER (Adelaide): Beyond Either/Or. The Pluralist Alternative in Herder's Thought . . . . . . . . . . . 53

JOCHEN JOHANNSEN (Witten/Herdecke): Vom Zeitigen in der Geschichte. Revolution, Zeiterfahrung und historische Sinnbildung beim späten Herder 71

Hans Peter Herrmann (Freiburg i. B.): „Mutter Vaterland «. Herders Historisierung des Germanenmythos und die Widersprüchlichkeit

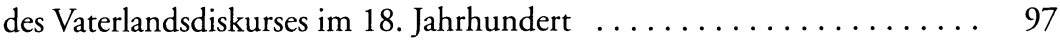

SAMSON B. KnOLL (Monterey, California): Europe in the History of Humanity. Herder, Kurt Breysig, and the Discourse on Eurocentrism in the Study of World History $\ldots \ldots \ldots \ldots \ldots \ldots \ldots \ldots \ldots \ldots \ldots \ldots \ldots \ldots \ldots \ldots$

\section{KONTROVERS}

Ulrike ZeUCH (Wolfenbüttel): Sentio, Ergo Sum. Herder's Concept of »Feeling " versus Kant's Concept of »Consciousness" . . . . . . . . 143

Karl Menges (Davis, California): "Sinn« and "Besonnenheit". The Meaning of "Meaning" in Herder

\section{REPLIK}

MichaEl Morton (Durham, North Carolina): Critical Realism and the "Critique of the Concept . . . . . . . . . . . . . . . . . . . . 177 


\section{BIBLIOGRAPHIE}

DORIS KUHLES (Weimar): Herder-Bibliographie 1995/1996 _. . . . . . . . . 191

Namen- und Personenregister zur Herder-Bibliographie . . . . . . . . . . . . 247

Das Herder-Jahrbuch und die Internationale Herder-Gesellschaft . . . . . . 254

Adressen der Autoren und Herausgeber . . . . . . . . . . . . . . . 256 


\section{VORWORT}

Die Beiträge zum vorliegenden, vierten Band des Herder-Jahrbuchs stammen aus verschiedenen Kontexten. Die ersten sechs Aufsätze - von John Zammito, Ralf Simon, Vicki Spencer, Jochen Johannsen, Hans Peter Herrmann und Samson B. Knoll wurden auf der Internationalen Herder-Tagung in Monterey zum Thema "Herder and History" präsentiert und für die Veröffentlichung überarbeitet. Samson Knoll war Gastgeber der Tagung, die im Oktober 1996 im Monterey Institute of International Studies stattfand - eine sorgfältig organisierte, gut abgestimmte (auch dem Leiter der Sprachabteilung des Instituts, Andreas Winkler, sei Dank!), sehr arbeitsintensive Tagung, von Peter Hanns Reill kundig mit seinem Eröffnungsvortrag eingeleitet und ein Jubiläum "in disguise«, denn: 1985 wurde die International Herder Society/Internationale Herder-Gesellschaft (IHS/IHG) ebendort, in Monterey, California, gegründet. Daß das elfjährige statt des zehnjährigen Jubiläums der IHS/IHG begangen` wurde, gehört zum liebenswert Unkonventionellen der Herder- Gesellschaft. Ein wunderbarer Empfang in Doc's Lab in (John Steinbecks) Cannery Row und ein traumhaftes Dinner im »Nepenthe" ("Quaff, oh, quaff this kind nepenthe", E. A. Poe) bei Sonnenuntergang am Pazifik gaben der Tagung den Rahmen, dem das Bild selbst, die Tagung, an Intensität in nichts nachstand. Alle Beiträge waren allen Teilnehmern und Teilnehmerinnen (aus Deutschland, Australien und den USA) vor der Tagung zugeschickt worden, so daß sich alle ausführlich mit den Texten auseinandersetzen konnten. Die Verfasser und Verfasserinnen der Beiträge hatten dann in Monterey kurz Gelegenheit zur thesenartigen Zusammenfassung, und es schloß sich eine bestens vorbereitete, ausführliche Diskussion an.

Die Sparten Kontrovers und Replik sind neu im Jahrbuch. Ihre Einrichtung bedeutet nicht, daß sie in jedem Band aktiviert werden müßten, sondern immer dann, wenn Aktivitäten in der Forschung es angezeigt sein lassen. Beide Rubriken sollen Angebot und Hinweis auf einen Ort für und eine Möglichkeit zum wissenschaftlichen Streitgespräch sein. Unter Kontrovers stehen Ulrike Zeuch und Karl Menges einander gegenüber, wie es sich traf auf der alljährlichen Sitzung der IHS/IHG im Rahmen der Jahrestagung der American Society for Eighteenth-Century Studies (ASECS) 1996 in Nashville, Tennessee. Die Sitzung trug den Arbeitstitel "Herder's Antikantianism«. Unter dem Rubrum Replik nimmt Michael Morton Gelegenheit zu Verteidigung und Widerwort gegen Robert Leventhal, der Morton im Herder Jahrbuch 1996 kritisiert hatte.

Die Textbeiträge decken ein breites Spektrum der internationalen Herder-Forschung $a b$, und nicht nur das. Es war für die Herausgeber ein Vergnügen zu sehen, auf welch hohem Reflexionsniveau diese Forschung betrieben und in welch breitem Kontext Herder situiert wird. Auffällig und gleichfalls erfreulich ist es, zu beobachten, wie die Herder-Forschung sowohl von aktuellen Problemlagen ausgeht, als auch pro- 
duktiv zu ihnen beiträgt. Wissenschaftsgeschichte, postmoderner Skeptizismus gegenüber Totalitätsentwürfen, logisch-analytische Fragestellungen, Legitimität des Geschichtlichen, moderne Nationalismusforschung, Entwürfe von Konzeptionen zur Weltgeschichte, Konzepte des Kognitivismus, Semantik und Systemtheorie sowie Skeptizismus-Kritik - alles das weist deutlich hin auf ein Herder-Verständnis und eine Auffassung vom 18. Jahrhundert, die die Fruchtbarkeit, Ergiebigkeit und Weite der Forschungsfelder herausstreichen. Erfreulich schließlich ist für die Herausgeber der hohe Anteil jüngerer Forscher und Forscherinnen im Feld der Dix-huitièmistes.

Beschlossen wird der Band mit der Herder-Bibliographie für die Berichtsjahre 1995 und 1996. Doris Kuhles von der Herzogin Anna Amalia Bibliothek in Weimar gibt auch dieses - zweite - Mal wieder unerläßliche Informationen zur Herder-Forschung an die Hand. Die Herausgeber begrüßen in diesem Zusammenhang die Fortführung der ausgezeichneten Kooperation mit der Stiftung Weimarer Klassik.

Wie dem Titelblatt zu entnehmen, ist Wilfried Malsch als Herausgeber des Jahrbuchs ausgeschieden. Er hat Band 1 als Mit-, die Bände 2 und 3 als Hauptherausgeber in Zusammenarbeit mit anderen erfrolgreich betreut. Für seine Arbeit sei ihm an dieser Stelle gedankt.

Ein Unternehmen wie das Herder-Jahrbuch ist ohne die Hilfe von Kollegen, Freunden und Institutionen nicht denkbar. Sehr zu danken für wertvolle Gutachtertätigkeit ist hier nachträglich Joachim Dyck (Oldenburg), Gerhard Sauder (Saarbrücken) und Jürgen Trabant (Berlin). Für Hilfestellung beim Zustandekommen des vorliegenden Bandes als Ratgeber und Gutachter danken wir insbesondere David Weberman (Madison, Wisconsin), Karl J. Fink und Norm Watt (beide Northfield, Minnesota), Tino Markworth (Stanford, California), Hugh B. Nisbet (Cambridge, UK), Wilhelm Schmidt-Biggemann und Jürgen Trabant (beide Berlin).

Samson B. Knoll, Hans Adler, Wulf Koepke 